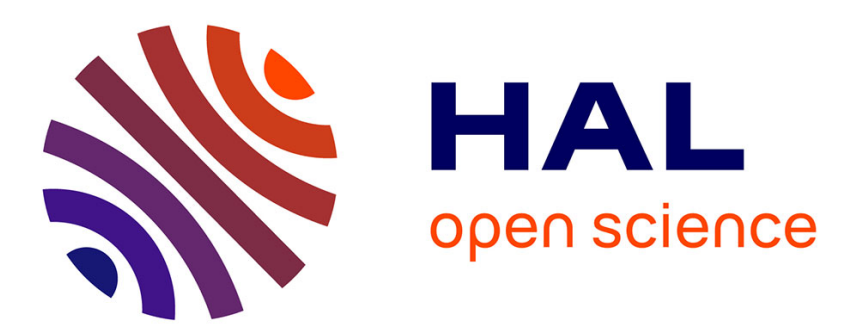

\title{
A novel representation method of non-ideal surface morphologies and its application in shaft-hole sealing simulation analysis
}

Jianshun Wu, Lihong Qiao, Zuowei Zhu, Nabil Anwer

\section{- To cite this version:}

Jianshun Wu, Lihong Qiao, Zuowei Zhu, Nabil Anwer. A novel representation method of nonideal surface morphologies and its application in shaft-hole sealing simulation analysis. Proceedings of the Institution of Mechanical Engineers, Part B: Journal of Engineering Manufacture, 2017, $10.1177 / 0954405417738284$. hal-01653817

\section{HAL Id: hal-01653817 https://hal.science/hal-01653817}

Submitted on 1 Dec 2017

HAL is a multi-disciplinary open access archive for the deposit and dissemination of scientific research documents, whether they are published or not. The documents may come from teaching and research institutions in France or abroad, or from public or private research centers.
L'archive ouverte pluridisciplinaire HAL, est destinée au dépôt et à la diffusion de documents scientifiques de niveau recherche, publiés ou non, émanant des établissements d'enseignement et de recherche français ou étrangers, des laboratoires publics ou privés. 


\title{
A novel representation method of non-ideal surface morphologies and its application in shaft- hole sealing simulation analysis Jianshun Wu', Lihong Qiao', Zuowei Zhu ${ }^{2}$, Nabil Anwer
}

\begin{abstract}
Assembly process simulation has been recognized as an effective tool for design verification. The representation of actual part surfaces produced by manufacturing processes is an important issue for assembly simulation. Manufactured part surfaces can also be regarded as non-ideal surface morphologies caused by manufacturing errors. This paper presents a new approach to describe non-ideal cylindrical surface morphologies. A deviation coordinate system is developed by adding a new deviation dimension along the normal direction of the nominal surface modelled in the cylindrical surface curvilinear coordinate system. Considering characteristics of the cylindrical surface machining process, a unified expression of combined Hermite polynomials and Fourier series is used to demonstrate variations that commonly appear on manufactured non-ideal cylindrical surfaces. The Hermite-Fourier polynomials constitute multimorphologies resulting from different manufacturing errors. In the proposed method, a parametric matrix is created from the expansion of the Hermite-Fourier polynomials. Each morphology can be represented by a corresponding matrix. The total deviation of a non-ideal part surface is the sum of deviations caused by each manufacturing error source through a linear combination of various matrices. The effectiveness of the proposed method is verified by a simulation of the sealing function of shaft-hole assemblies.
\end{abstract}

\section{Keywords}

Non-ideal surface morphology representation; Deviation coordinate system; Hermite-Fourier polynomials; Parametric matrix; Sealing simulation; Manufacturing errors.

\section{Introduction}

In the assembly process, the performance of two mating components depends largely on the contact status and clearance between their mating surfaces. As a critical part of product design, assembly simulation provides important information for evaluation of product performance. However, at present, most available simulation systems for assembly analysis are based on the ideal part geometry, while ignoring the possible deviations on part surfaces that may cause assembly failure. Therefore, it is necessary to incorporate the manufactured part surfaces in the assembly simulation model to consider actual product geometry..
With this regard, efficient methods to model practical manufactured geometry of cylindrical surfaces should be developed. With the help of such methods, the impact of various manufacturing errors on the cylindrical surfaces

\footnotetext{
${ }^{1}$ Department of Industrial and Manufacturing Systems Engineering, Beihang University, China ${ }^{2}$ Laboratoire Universitaire de Recherche en Production Automatisee, Ecole Normale Superieure de Paris Saclay, France

Corresponding author:

Lihong Qiao, Department of Industrial and Manufacturing Systems Engineering, Beihang University, 100191, Beijing, China.

Email:lhqiao@buaa.edu.cn
} 
could be analyzed accurately and consistently.

The modeling of manufactured surface has received much research attention. Some previous studies lack of considering form deviations in the modeling of the non-ideal surfaces. Louhichi et al. modelled the position and orientation defects of components based on the shape of the tolerance zone, the toleranced feature type and the tolerance type ${ }^{1}$. Dantan and Qureshi utilized convex hulls defined in the parametric space to simulate the influences of geometric deviations on the geometrical behavior of the mechanism ${ }^{2}$. However, these studies are lacking in the consideration of form deviations, which have a great impact on the functional and performance of products and should be modelled. Anwer et al. investigated the fundamentals of skin models at a conceptual, geometric and computational level to address the geometrical variations ${ }^{3,4}$. Schleich and Anwer proposed the skin model shapes paradigm by the superposition of the nominal surface and geometric deviations that are divided into systematic deviations and random deviations ${ }^{5,6}$. This paradigm provides a method for the modeling, representation and analysis of geometric deviations. A method proposed by Zhu et al. improved the pre-processing stage to prepare the skin model shapes to be used for tolerance analysis ${ }^{7}$. Samper proposed to parameterize form deviations by building a geometrical model based on the natural modal shapes of the ideal surface, which simplifies the consideration of surfaces with form defects in assembly simulation ${ }^{8}$. With the application of the discrete modal decomposition, a surface with multi-scale deviations was simulated and evaluated with respect to tolerance specification by $\mathrm{Cao}$ et $\mathrm{al}^{9}$. Huang et al. developed a modebased method to characterize part geometric error using Discrete Cosine Transform $(\mathrm{DCT})^{10}$, in which the modes related to the manufacturing process error could be identified. Based on the measurement data, a manufacturing signature model was established by XXX to facilitate the representation of the actual profiles of manufactured surfaces 11, 12. With the manufacturing signature and the assembly conditions, Corrado, Polini and Moroni proposed a geometric model to simulate the assembly of parts with geometric deviations, based on which a new CAT (Computer Aided Tolerancing) tool was developed ${ }^{13}$. To generate consistent skin model shapes, two deviation combination methods were proposed to preserve the deviations of surfaces by Yan and Ballu ${ }^{14}$. Cai studied the rigid and compliant variation modeling of sheet metal assembly ${ }^{15,16}$. The assembly sequence planning research are also be studied ${ }^{17-19}$.

Meanwhile, the modeling of cylindrical surface deviations has also drawn research interest. A typical method is the combination of the Fourier series and Chebyshev or Legendre polynomials, which has been employed to model cylindricity errors. Zhang et al. introduced a unified functional tolerancing approach involving Legendre and Fourier polynomials for modeling and characterizing typical geometrical errors commonly found in machined cylindrical parts ${ }^{20}$. Additionally, Ni and Yao attempted to model the cylindricity errors with Legendre/Fourier polynominals and the effects of the cylindricity error on position accuracy are analyzed ${ }^{21}$. Henke et al. put forward two methods to model the cylindricity errors, one based on Chebyshev/Fourier polynomials and the other based on a linear combination of Eigen shapes derived directly from the measurement data ${ }^{22}$.

Since cylindrical features are basic geometric features that have been widely used in assembly, we will put our focus on cylindrical features in the paper. The effects of different manufacturing errors on the cylindrical features are studied. Zhang et al. studied the typical manufacturing errors, such as errors caused by spindle rotation, fixture distortion, misalignment of spindles and work centers, workpiece deflection et al. The effects of these errors on manufactured surfaces are also investigated ${ }^{20}$. Muhummad et al. described a concurrent measurement of radial, axial and angular motions of spindle using concentric circle grating and phase modulation interferometers in order to depict the deviation of the manufactured surface from the nominal surface $^{23}$. Chien expanded the approximated harmonic model by adding the knowledge of machining process ${ }^{24}$. It was also concluded that the number of lobes on manufactured surface is related to the number of jaws on the chuck which holds the workpiece ${ }^{25,26}$. A cutting force model was built to analyze the workpiece deflection caused by the cutting force ${ }^{27,28}$. The research of XXX also established a relationship between cutting time and tool wear and found it linear in the nominal wear stage ${ }^{29}$. A relationship between the actual and nominal depth of cut was also revealed $^{30}$.

Many of the proposed models represent the manufactured surface either by discrete surface points or by the whole surface shape. A model that can represent the deviated surface with respect to its ideal design remains to be developed. It is 
necessary to develop a precise non-ideal surface morphology representation method for highfidelity simulation modeling. The representation should consider the manufacturing errors that affect the final manufactured surface morphology. This paper proposes a deviation coordinate system and Hermite-Fourier combination polynomials to express manufactured cylindrical part surface morphologies. The deviations of multiple morphologies of a manufactured cylindrical surface can be easily calculated by the parametric matrices resulting from the expansion of the polynomials. The effectiveness of the method is verified through simulation and analysis of the sealing function of shaft-hole assemblies.

\section{Coordinate representation and formation of the non-ideal cylindrical surface}

The nominal cylindrical surface in cylindrical coordinate system

Due to the extensive application of cylindrical surfaces in product design, it is significant to establish an effective coordinate representation for cylindrical surfaces. For cylindrical surfaces, a cylindrical coordinate system $(r, z, \theta)$ is commonly used to describe the ideal (i.e. designed and intended) geometry, namely the nominal surface. In this coordinate system, the nominal surface can be expressed as:

$$
r=f(z, \theta)=r_{0} \quad 0 \leq \mathrm{z} \leq z_{n}, 0 \leq \theta \leq 2 \pi
$$

The function represents a nominal cylindrical surface in a $3 \mathrm{D}$ coordinate system, where $r_{0}$ is the radius of the nominal cylinder and $z_{n}$ is the height of the cylinder. Fig. 1 shows the nominal cylindrical surface in the cylindrical coordinate system.

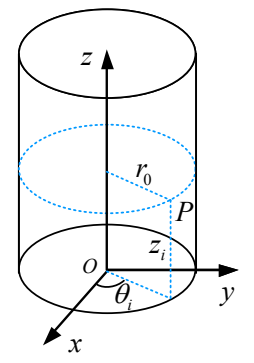

Figure $1 \mathrm{~A}$ nominal cylindrical surface in cylindrical coordinate system

Point $\mathrm{P}$ could be expressed as:

$$
r=r_{0}, \mathrm{Z}=z_{i}, \theta=\theta_{i}
$$

Where $\mathrm{i}=1,2,3 \quad \mathrm{n}, z_{i}$ is the height of point $P$, and $\theta_{i}$ is the circumferential position of $P$. Any point on the nominal surface can be represented by $r_{0}, z_{i}$ and $\theta_{i}$. With the definition of the cylindrical coordinate, the non-ideal cylindrical surface can be easily described.

\section{Relationship between non-ideal cylindrical surfaces and nominal surfaces}

The performance of an assembled product is associated with the shape of mating component surfaces in the assembly. It is important to consider the effects of the manufactured shape in the simulation analysis to achieve more practical and accurate understanding of the assembly performance.

For example, Fig. 2 illustrates different nonideal shaft-hole assemblies. In this figure, the hole is nominal, while the shaft is non-ideal and three non-ideal surface shapes are included in the assemblies. It should be noted that various nonideal surface shapes that comply with the same tolerance zone $T z$ may have different effects on the sealing performance of the assembly.

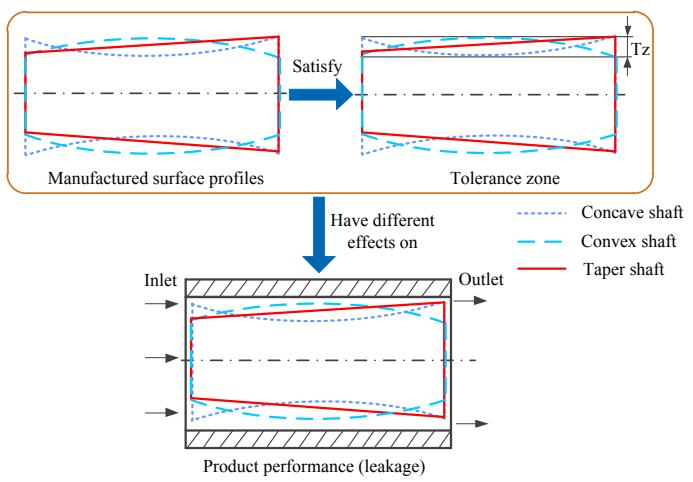

Figure 2 Illustration of the effect of different surface morphologies on product performance

It is obvious from Fig. 2 that, the product performance varies significantly when the shape of the manufactured surface changes. Therefore, in order to capture this variability, the relationship between the non-ideal manufactured surface and the nominal surface should be studied.

The manufactured non-ideal surface can be considered as a deviated state of its nominal geometry or ideal surface. Any point on the nonideal surface can be corresponded to a point on the nominal surface, and be defined as a "deviation" of that point from its theoretical position. As shown in Fig.3, the non-ideal surface resembles a blanket that wraps the nominal surface, with the thickness at each point of the blanket as the corresponding deviation of 
that point. Thus the non-ideal surface can be represented mathematically by the superposition of the nominal surface and deviations.

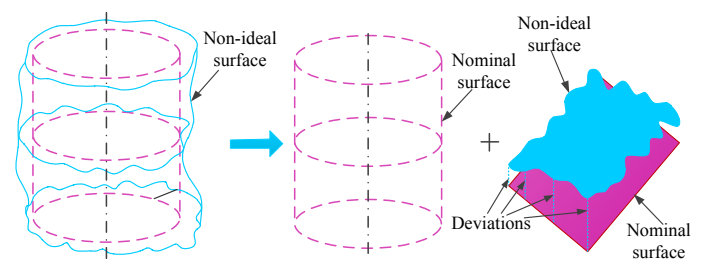

Figure 3 Relationship between non-ideal and nominal cylindrical surfaces

With this relationship, the non-ideal cylindrical surface can also be represented in the cylindrical coordinate system $(r, z, \theta)$, and three definitions are proposed as follows to describe the relationship.

Definition 1: According to the definition of the nominal surface in the cylindrical coordinate system, a two-dimensional system - cylindrical surface curvilinear coordinate system $(z, \theta)$ is defined $^{31}$. The entire nominal surface could be mapped onto this coordinate system.

In fact, the non-ideal surfaces could still be represented using cylindrical coordinate system $(r, z, \theta)$. Which is similar to the representation of an object (such as a satellite) in the geodetic coordinate system $(\varphi, \lambda, h)^{32}$, where $\varphi, \lambda$ and $h$ show the values of geodetic latitude, geodetic longitude, and geodetic height, respectively. The radius vector $r_{i}$ of a point $P^{\prime}$ on the non-ideal surface is the function of two coordinates $z_{i}$ and $\theta_{i}$. And it can actually be expressed in terms of $(r, z, \theta)$, that is:

$$
r_{i}=f\left(z_{i}, \theta_{i}\right)
$$

Definition 2: A variable $\delta$ can be defined as "deviation variable", and expressed as:

$$
\begin{gathered}
\delta_{i}=r_{i}-r_{0}=\varphi\left(z_{i}, \theta_{i}\right) \\
\delta=r-r_{0}=f(z, \theta)
\end{gathered}
$$

Equation (4), termed as "deviation function", is a function in the cylindrical surface curvilinear coordinate system.

According to Definition 1, the deviation variable $\delta_{i}$ represents the deviation of the nonideal surface points when $z=z_{i}$ and $\theta=\theta_{i}$. The deviation variable $\delta_{i}$ can also be regarded as an attribute of the point on the non-ideal surface in the cylindrical surface curvilinear coordinate $\operatorname{system}(z, \theta)$.

Definition 3: Based on the cylindrical surface curvilinear coordinate system $(z, \theta)$, by adding a deviation dimension, a novel 3D coordinate system - deviation coordinate system is defined as $(\delta, z, \theta)$.

It is known from Definition 2 that the non-ideal surface can be represented by one function in the deviation coordinate system. Therefore, it can be treated as a surface in the deviation coordinate system.

\section{The non-ideal cylindrical surface in deviation coordinate system}

According to Definition 2 and 3, the deviation $\delta$ could be regarded as a vector: the direction of $\delta$ is the radial direction of the cylindrical surface; and the value of $\delta$ is the distance between the point on the nominal surface and its corresponding point on the non-ideal surface. Since directions of $\delta, z$ and $\theta$ are orthogonal, the $3 \mathrm{D}$ deviation coordinate system $(\delta, z, \theta)$ constructed by $\delta, z$ and $\theta$ is an orthogonal coordinate system. In the deviation coordinate system, the origin of the deviation dimension is no longer the same for each point. For point $P^{\prime}$ on the non-ideal surface, the origin of the corresponding deviation coordinate is point $P$ on the nominal surface, as shown in Fig. 4. The figure gives a cross-section of the cylinder when $z=z_{i}$.

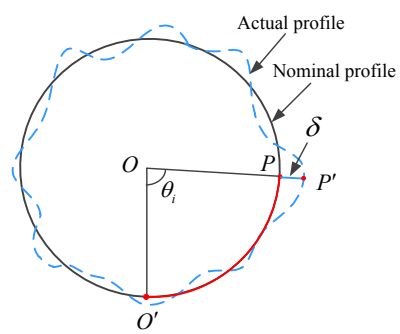

Figure 4 Representation of non-ideal surface in deviation coordinate system

In the figure, $O$ is the center of the nominal profile. The cylindrical surface curvilinear coordinate system is established on the nominal surface and $O^{\prime}$ is the origin of the $\theta$ coordinate.

According to the definition of the deviation coordinate system, every point can be represented by the deviation coordinates in this coordinate system. And the deviation function established in this coordinate system has the following characteristics:

a) If $\delta \equiv 0$ for all the points, the non-ideal cylindrical surface yields to its nominal and there is no deviation; 
Wu et al.

b) If $\delta=d$ for all the points and $d$ is a constant, then the non-ideal cylindrical surface has the same geometrical shape as the nominal surface thus only a dimensional deviation exists;

c) The distribution of the deviations can be plotted by the deviation function.

Such a definition of the deviation coordinate system provides a clear and easy representation of non-ideal surfaces. The non-ideal surface could be cast into a standard and unified form by the deviation function, which leads to simplified modeling and calculation of the non-ideal surfaces in computer-aided systems.

\section{Formation of the non-ideal surface} morphology

In a manufacturing process, the manufactured surface of the workpiece is not consistent with the nominal surface due to manufacturing errors. Under the influence of some typical errors caused by cutting force, tool wear, thermal factors, spindle motion error and fixture error, surface morphologies are introduced. Table 1 provides a description of the manufacturing errors as well as their corresponding non-ideal surface morphology and mathematical formulation. 
Table 1 Description of typical manufacturing errors in the turning process and mathematical formulation of corresponding non-ideal surface morphologies

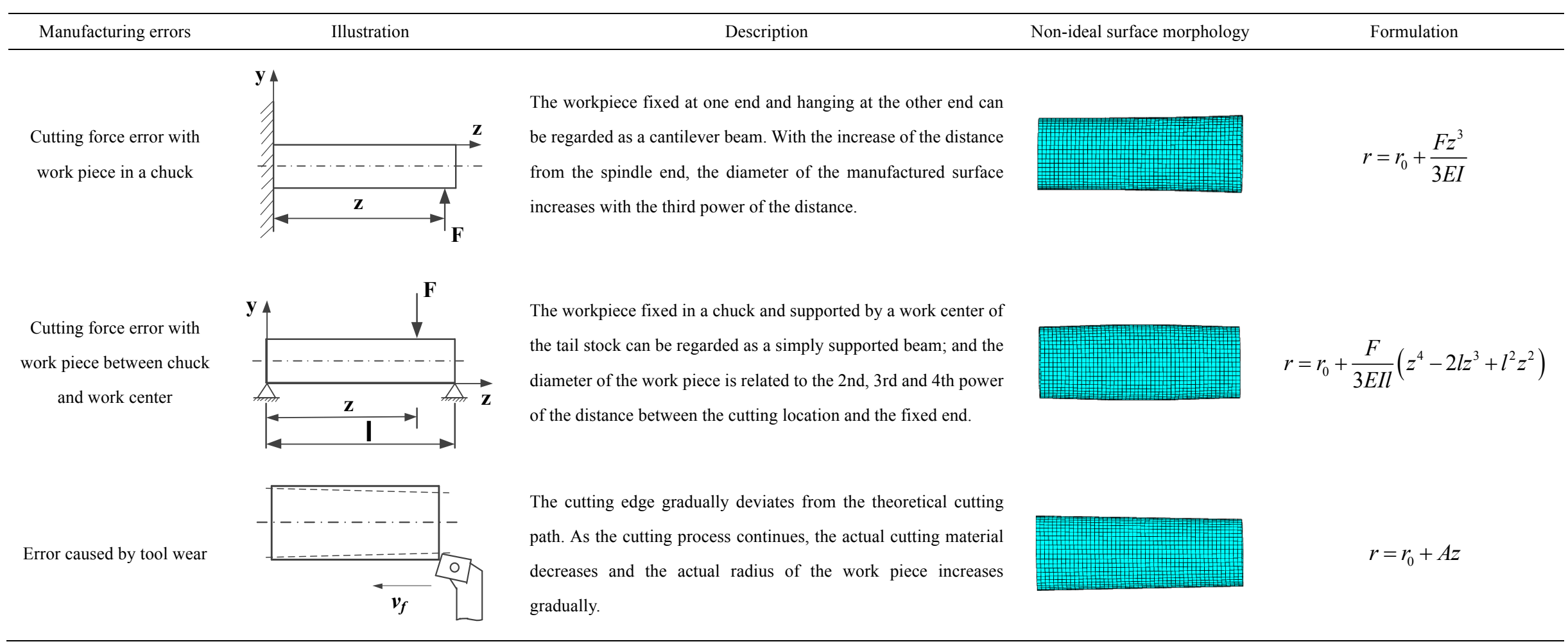




\section{Deviation modeling and parametric representation of the non-ideal cylindrical surface}

\section{Deviation modeling of the non-ideal surface}

Two motions exist in a cylindrical turning process, namely main motion and feed motion as depicted in Fig. 5. The main motion is a rotational motion of the spindle. The variations of spindle will result in the variation of the relative position between the workpiece and tool. The deviation on the manufactured surface resulting from the main motion is only related to the angle of rotation, and is therefore periodic.

Feed motion is the translation of the tool along the axis of the spindle. Deviations may occur due to force deformation caused by the equipment and cutting force and the variation of the tool tip geometry caused by heat and/or tool wear. Such deviations are generally dependent on the axial position, while unaffected by the rotation of the workpiece. The deviations of the manufactured surface during feed motion can be expressed by a function of the distance between the cutting location and the spindle end.

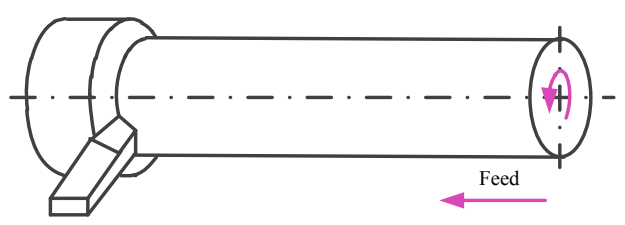

Figure $\mathbf{5}$ Two motions in cylindrical turning process

A manufactured cylindrical surface is composed of multiple non-ideal surface morphologies, each corresponding to a certain manufacturing error. Each morphology includes deviations both in the axial direction and along the circumferential direction, the former can be represented by polynomials and the latter is periodic.

Chebyshev and Legendre polynomials ${ }^{20,} 22$ were adopted to demonstrate the deviations along the axial directions. Since the range of Chebyshev and Legendre polynomials is $[-1,+1]$, the axial coordinate should be mapped to $[-1,+1]$ first when representing the cylindrical surface. Different from the aforementioned polynomials, the range of a Hermite polynomial is $(-\infty,+\infty)$, which makes it easier to describe the cylindrical surface with this polynomial.

Thus, Hermite polynomials and Fourier series can be used respectively to express the deviations along these two directions. The comprehensive expression of the cylindrical non-ideal surface deviations caused by multiple errors can be expressed by the combination of the Hermite polynomials and Fourier series, as shown in Eq. 5.

$\delta=\sum_{i=0}^{u} H_{i}(z)\left[\sum_{j=0}^{v} a_{i j} \cos (j \theta)+\sum_{j=0}^{v} b_{i j} \sin (j \theta)\right](5)$

Where $\delta$ is the deviation of the non-ideal surface, $r_{0}$ is the nominal radius of the cylinder, $u$ is the order of the Hermite polynomials, $v$ is the order of Fourier series, $a_{i j}$ and $b_{i j}$ are coefficients of Fourier series. Equation (5) is named "Hermite-Fourier polynomials" in this study.

The Hermite polynomial sets $\left\{H_{n}(z)\right\}$ are orthogonal and defined in $(-\infty,+\infty)$ with weight. According to its definition,

$$
H_{n}(z)=(-1)^{n} e^{z^{2}} \frac{d^{n}\left(e^{-z^{2}}\right)}{d z^{n}} \quad(n=0,1, \ldots)
$$

The first five Hermite polynomials are:

$$
\begin{gathered}
H_{0}(z)=1 \\
H_{1}(z)=2 z \\
H_{2}(z)=4 z^{2}-2 \\
H_{3}(z)=8 z^{3}-12 z \\
H_{4}(z)=16 z^{4}-48 z^{2}+12
\end{gathered}
$$

Each morphology is a linear combination of several Hermite polynomials.

The selection of these two orthogonal functions conforms to the motion characteristics of the turning or grinding process. The orthogonality of these two polynomials facilitates the expression of the non-ideal surface.

From the above analysis, the Hermite-Fourier polynomials of typical non-ideal surface morphologies can be concluded in Table 2 . 
Wu et al.

\begin{tabular}{|c|c|c|c|}
\hline No. & Manufacturing errors & Non-ideal surface morphology & Mathematical formulation \\
\hline 1 & $\begin{array}{l}\text { Cutting force error with } \\
\text { workpiece in a chuck }\end{array}$ & & $\delta=A H_{1}(z)+B H_{3}(z)$ \\
\hline 2 & $\begin{array}{c}\text { Cutting force error with } \\
\text { workpiece between chuck and } \\
\text { work center }\end{array}$ & 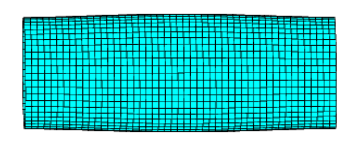 & $\begin{array}{l}\delta=A H_{0}(z)+B H_{1}(z)+C H_{2}(z) \\
+D H_{3}(z)+E H_{4}(z)\end{array}$ \\
\hline 3 & Error caused by tool wear & & $\delta=A H_{0}(z)+B H_{1}(z)$ \\
\hline 4 & $\begin{array}{c}\text { Error caused by thermal factors } \\
\text { during cutting }\end{array}$ & & $\delta=A H_{0}(z)+B H_{1}(z)$ \\
\hline 5 & Spindle motion error & & $\cdot\left[\sum_{j=0}^{j=\infty} a_{i j} \cos k \theta+\sum_{j=0}^{j=\infty} b_{i j} \sin k \theta\right]$ \\
\hline 6 & Fixture error & & $\delta=\left(A H_{0}(z)+B H_{1}(z)\right) \cos 3 \theta$ \\
\hline
\end{tabular}

In Table 2, A, B, C, D and E are constants. Therefore, the non-ideal surface morphology generated by each manufacturing error can be represented by Hermite-Fourier polynomials, which allows the unified expression of various deviation functions. Different non-ideal surface morphologies have their characteristic parameters, and these parameters can be conveniently expressed by a parametric matrix.

\section{Parametric representation of the non-ideal surface}

Establishment of the parametric matrix for non-ideal surface manufacturing conditions, the expression of the manufactured surface should combine multiple morphologies resulting from different manufacturing errors. By analyzing the non-ideal cylindrical surface morphology and the characteristics of the Hermite-Fourier polynomials, a parametric matrix can be created from the expansion of the Hermite-Fourier polynomials. Using the polynomials in Eq. 5, the deviation function can be represented as a multiplication of three matrices:

\section{Considering the differences between}

$$
\delta^{k}=\left[\begin{array}{llll}
H_{0} & H_{1} H_{2} & H_{I}
\end{array}\right]\left[\begin{array}{ccccccc}
a_{0,0} & a_{0,1} & b_{0,1} & a_{0,2} & b_{0,2} & a_{0, J} & b_{0, J} \\
a_{1,0} & a_{1,1} & b_{1,1} & a_{1,2} & b_{1,2} & a_{1, J} & b_{1, J} \\
a_{i-1,0} & a_{i-1,1} & b_{i-1,1} & a_{i-1,2} & b_{i-1,2} & a_{I, J} & b_{I, J}
\end{array}\right]\left[\begin{array}{c}
1 \\
\cos \theta \\
\sin \theta \\
\cos 2 \theta \\
\sin 2 \theta \\
\cos (J) \theta \\
\sin (J) \theta
\end{array}\right]
$$

The matrix in the middle is the parametric matrix of the Hermite-Fourier polynomials. In 
this matrix, $i=1,2,3 \quad I, j=1,2,3 \quad \mathrm{~J}$, which represent items of the Hermite polynomial and Fourier series respectively. The $\mathrm{k}$ in $\delta^{k}$ represents a certain manufacturing error. High order terms of the Hermite-Fourier polynomials have less impact on the non-ideal surface and can be omitted when studying the large-scale deviations of the manufactured surface. Thus the parametric matrix in the deviation function can be simplified as a $5 * 7$ matrix shown in Eq. 9.

$$
\delta^{k}=\left[\begin{array}{lllll}
H_{0} & H_{1} & H_{2} & H_{3} & H_{4}
\end{array}\right]\left[\begin{array}{lllllll}
a_{0,0} & a_{0,1} & b_{0,1} & a_{0,2} & b_{0,2} & a_{0,3} & b_{0,3} \\
a_{1,0} & a_{1,1} & b_{1,1} & a_{1,2} & b_{1,2} & a_{1,3} & b_{1,3} \\
a_{2,0} & a_{2,1} & b_{2,1} & a_{2,2} & b_{2,2} & a_{2,3} & b_{2,3} \\
a_{3,0} & a_{3,1} & b_{3,1} & a_{3,2} & b_{3,2} & a_{3,3} & b_{3,3} \\
a_{4,0} & a_{4,1} & b_{4,1} & a_{4,2} & b_{4,2} & a_{4,3} & b_{4,3}
\end{array}\right]\left[\begin{array}{c}
1 \\
\cos \theta \\
\sin \theta \\
\cos 2 \theta \\
\sin 2 \theta \\
\cos 3 \theta \\
\sin 3 \theta
\end{array}\right]
$$

The parameters in the matrix are relevant to a certain morphology and are determined by the corresponding manufacturing error. The parameter values reflect the extent of variation of the morphology. Take the first manufacturing error in Table 3 as an example, according to the non-ideal surface morphology formulation defined in Table 1 and Table 2, the deviation of the manufactured surface is only related to the third power of the distance between the cutting location and the spindle end. The parameter $a_{3,0}$ is the coefficient of Hermite polynomial $H_{3}(z)$, however, $H_{3}(z)$ contains not only the cubic polynomial but also a linear polynomial. In order to compensate for the effects of the linear polynomial, $H_{1}(z)$ is introduced with a coefficient $a_{1,0}$, the rest of the parameters in the matrix are 0 . The other parametric matrices in Table 3 are calculated in a similar way. Table 3 summarizes the parametric matrix of each morphology caused by a certain manufacturing error.

Table 3 Hermite-Fourier parametric matrix of each non-ideal surface morphology caused by a certain error

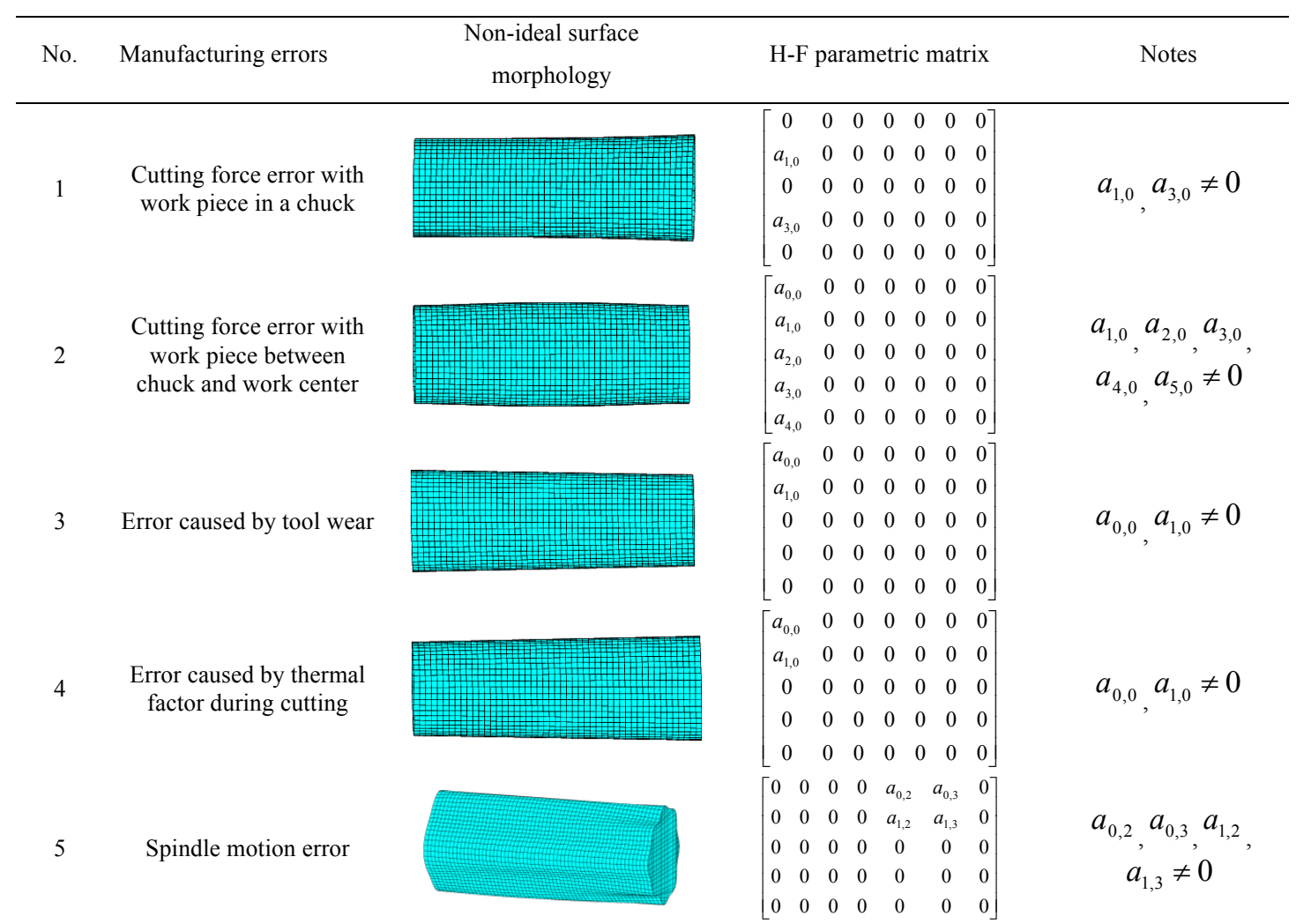


Wu et al.

6 Fixture error $\quad$ (t)

Computing the total deviations for the non-ideal surface

The total deviations of the non-ideal surfaces are the consequence of various manufacturing errors. Each error plays a definite role in the manufacturing process. Considering the independence of the manufacturing errors during the manufacturing process, the total deviations of the manufactured surface can be regarded as the composition of the deviations caused by each error. With the unified expression of each nonideal surface morphology, the superposition of multiple Hermite-Fourier polynomials is still a Hermite-Fourier polynomial. The total deviations of the manufactured surface can be considered as the superposition of corresponding parametric matrices. The expression of total deviations of a manufactured surface is given in Fig. 6.

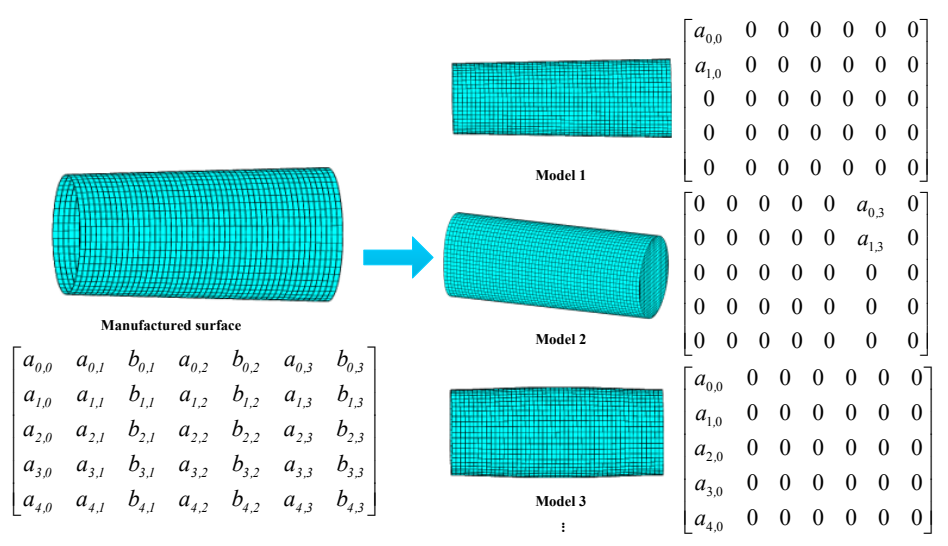

Figure 6 Expression of total deviations of a manufactured surface

Fig.6 shows a manufactured surface affected by several errors, in which tool wear is the dominant factor, and the expression of the surface consists of Model 1, Model 2 and Model 3 et al. Every model is a non-ideal surface morphology caused by one manufacturing error.

On account of the unified representation of each non-ideal surface morphology and the different levels of the impact caused by manufacturing errors, the manufactured surface can be modeled by the linear combination of the non-ideal surface morphologies by parametric matrices.

\section{Verification of the representation method of non-ideal cylindrical surface}

To verify the effectiveness of the representation method, a shaft-hole assembly is selected in the following case study. Using the Herimite-Fourier polynomials, several types of shaft-hole assembly are modeled, then, sealing simulations and subsequent analysis are performed.
Three typical non-ideal long shaft cylindrical surfaces (taper, convex, concave) affected by three different primary manufacturing errors are chosen for sealing simulation. For taper surface, tool wear is the dominant error during manufacturing. For a low-rigidity workpiece clamped with a chuck and work center, the convex shape will appear under the effect of the cutting force, and the concave surface will be resulted from the vertical misalignment of chuck and work piece center. Models of these three features are shown in Fig. 7.

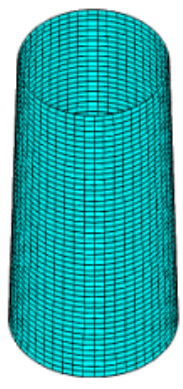

(a) Taper

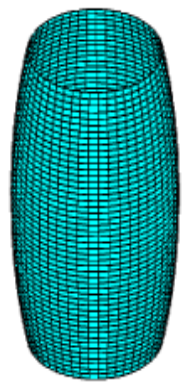

(b) Convex

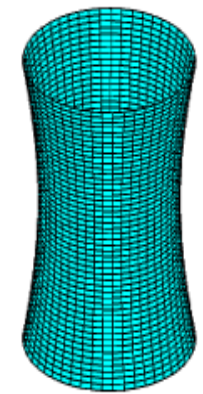

(c) Concave
Figure 7 Three typical non-ideal surfaces in turning process 
With these three non-ideal shaft surfaces, the assembly structures of non-ideal long shafts and nominal holes are constructed. And the clearance fit is applied to these assemblies. Exemplary shaft-hole assemblies for sealing simulation analysis are given in Fig. 8.

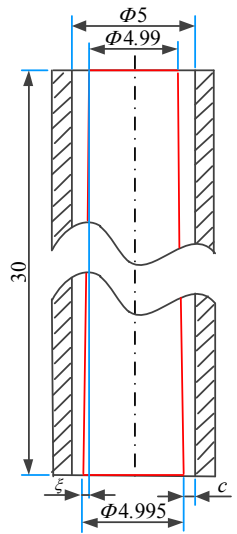

(a) Taper-Ideal

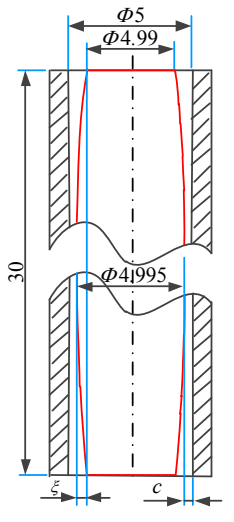

(b) Convex-Ideal

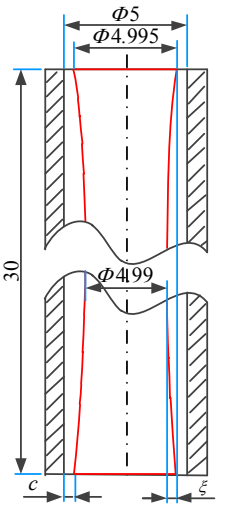

(c) Concave-Ideal
Figure 8 Example shaft-hole assemblies

In the figure, $c$ denotes the clearance of the hole and shaft, $\xi$ is the form variation of the nonideal surface shape. The values of $\xi$ vary during the simulation. With these two parameters, the influence of geometrical tolerances on the sealing requirement of the mating structure will be estimated, which facilitates the improvement and optimization of the product design.

The long shaft-hole examples used in the sealing simulation and analysis consist of several non-ideal surface morphologies. The tapered shaft is affected mainly by cutting tool wear error, along with other two non-ideal cylindrical surface morphologies caused by cutting force and thermal effect. According to the mathematical formulations and parametric matrices listed in Table 1, Table 2 and Table 3, when $\xi=0.0004$ $\mathrm{mm}$, the parameters $a_{0,0}$ and $a_{1,0}$ in the third matrix listed in Table 3 are $4 \times 10^{-4}$ and $-6.667 \times 10^{-6}$, and the variables $a_{1,0}$ and $a_{3,0}$ in the first matrix are $1.389 \times 10^{-9}$ and $2.315 \times 10^{-10}$, the parameters $a_{0,0}$ and $a_{1,0}$ in the fourth matrix are $5 \times 10^{-5}$ and $-8.333 \times 10^{-7}$. Similarly, the other surfaces applied to sealing simulations are modeled following the same principle.

The preconditions are set for the simulation, in which the maximum dimension of shaft is 4.995 $\mathrm{mm}$ and $c=0.0025 \mathrm{~mm}$. The instances in Fig. 8 are applied to the sealing simulation analyses and the simulation is implemented in Fluent 6.3 following the general process of fluid analysis in the system. The parameter settings of the fluid in the simulation are listed in Table 4. The inlet pressure is $0.12 \mathrm{MPa}$, and the outlet pressure is barometric pressure. The leakage results are depicted in Fig. 9.

Table 4 Parameter settings of the fluid in sealing simulation

\begin{tabular}{cccccc}
\hline $\begin{array}{c}\text { Temperature } \\
\mathrm{K}\end{array}$ & $\begin{array}{c}\text { Viscosity } \\
\mathrm{kg} /(\mathrm{m} * \mathrm{~s})\end{array}$ & $\begin{array}{c}\text { Density } \\
\mathrm{k} / \mathrm{m} 3\end{array}$ & $\begin{array}{c}\text { Specific heat } \\
\mathrm{J} /(\mathrm{kg} * \mathrm{~K})\end{array}$ & $\begin{array}{c}\text { Thermal conductivity } \\
\mathrm{W} /(\mathrm{m} * \mathrm{~K})\end{array}$ & Flow characteristic \\
\hline 293.15 & 1.06 & 889 & 1845 & 0.145 & laminar \\
\hline
\end{tabular}

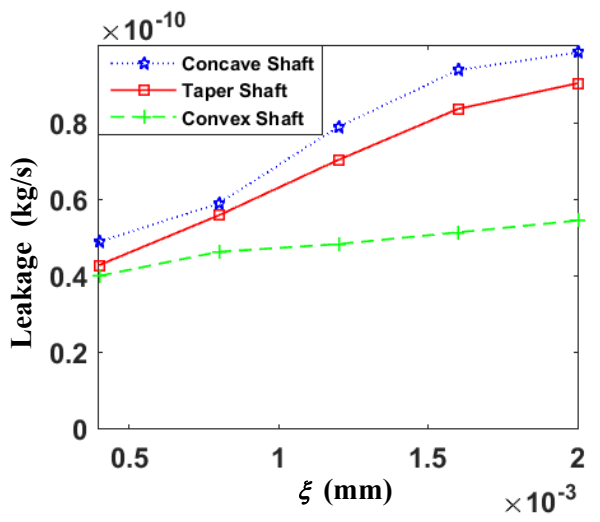

Figure 9 Comparison of leakage of different surface shapes
From Fig. 9, it should be noted that the leakage variation increases along with the increase of the form variation. The leakage variations are multitudinous because of the differences between surface shapes. By comparing the leakage of different surface shapes, it can be concluded that the leakage of convex shaft is minimum, the leakage of taper shaft is larger than convex shaft, and the leakage of concave shaft is maximum. The results also indicate that different non-ideal surface shapes that conform to the same tolerance zone have various effects on the functionality and performance of the product. Through this analysis, the validity of the representation method is verified by comparing the differences between 
Wu et al.

these shafts applied in the sealing simulation.

When implanting multiple simulations, the non-ideal cylindrical surfaces need to be added in the simulation model continually, therefore, they should be sustained and generated rapidly. Since the non-ideal surfaces are caused by multiple manufacturing errors, the parametric matrices proposed in this paper make it possible to consider each of the errors in the simulation. Moreover, the non-ideal surface can be easily obtained by changing the parameters in the parametric matrices when errors vary during manufacturing processes. The parameters in the matrix also represent different levels of effects of each error on the manufactured surface.

Unlike previous models established for analysis of product performance, the models developed by this representation method are based on non-ideal assemblies. To a large extent, the analysis results approximate the real conditions of the manufactured product. The representation method also facilitates the generation and application of non-ideal surfaces.

\section{Conclusions}

In this paper, a novel representation method for non-ideal surfaces is proposed and verified by the sealing simulation analysis of shaft-hole assemblies. A deviation coordinate system has been developed to describe the deviations of the non-ideal cylindrical surface. The system is established by adding a deviation dimension along the normal vector of the nominal surface expressed by a cylindrical surface curvilinear coordinate system, which makes the description of deviations more simplified and effective.

A unified formula is selected to express the deviations of the non-ideal cylindrical surfaces. The formula combines Hermite polynomials and Fourier series, representing deviations in the axial direction and the circumferential direction respectively. The parametric matrices created from the expansion of the Hermite-Fourier polynomials are utilized to represent the corresponding morphologies generated by the manufacturing errors. The parameters in each matrix correspond to the effects of one particular manufacturing error. The total deviations of the manufactured surface can be considered as the superposition of corresponding parametric matrices. The proposed representation method facilitates modeling of the non-ideal cylindrical surfaces for simulation process, and other application cases.

\section{Declaration of conflicting interests}

The authors declare that there is no conflict of interest.

\section{Funding}

This work is supported by the National Science Foundation of China (Grant 51575031) and National High-Tech. R\&D Program of China (No.2015AA043702). The authors would also like to thank Beijing Municipal Education Commission (Build a Project) for its support.

\section{References}

1. Louhichi B, Tlija M, Benamara A, et al. An algorithm for CAD tolerancing integration: Generation of assembly configurations according to dimensional and geometrical tolerances. Comput-Aided Des. 2015; 62: 259274

2. Dantan JY and Qureshi AJ. Worst-case and statistical tolerance analysis based on quantified constraint satisfaction problems and Monte Carlo simulation. Comput-Aided Des. 2009; 41: 1-12.

3. Anwer N, Ballu A, Mathieu L. The skin model, a comprehensive geometric model for engineering design. CIRP Ann - Manuf Technol. 2013; 62(1): 143-146

4. Zhang M, Anwer N, Stockinger A, et al. Discrete shape modeling for skin model representation. Proc Inst Mech Eng Part B-J Eng Manuf. 2013; 227(5): 672-680.

5. Schleich B, Anwer N, Mathieu L et al. Skin model shapes: A new paradigm shift for geometric variations modelling in mechanical engineering. Comput-Aided Des. 2014; 50: 115

6. Schleich B, Wartzack S. Approaches for the assembly simulation of skin model shapes. Comput-Aided Des. 2015; 65: 18-33

7. Zhu Z, Qiao L, Anwer N. An Improved Tolerance Analysis Method Based on Skin Model Shapes of Planar Parts. Procedia CIRP. 2016; 56:237-242.

8.Samper S, Adragna P A, Favreliere $\mathrm{H}$ et al. Modeling of 2D and 3D Assemblies Taking Into Account Form Errors of Plane Surfaces. J Comput Inf Sci Eng. 2010; 9: 790-792

9. Cao Y, Li B, Ye X, et al. Geometrical Simulation of Multiscale Toleranced Surface With Consideration of the Tolerancing Principle. J Comput Inf Sci Eng. 2015; 15(2): 021006

10. Huang W, Ceglarek D. Mode-based Decomposition of Part Form Error by DiscreteCosine-Transform with Implementation to 
Assembly and Stamping System with Compliant Parts. CIRP Ann-Manuf Technol. 2002; 51: 21-26

11. Colosimo B M, Moroni G, Petro S, et al. Manufacturing signature of turned circular profiles. Proceedings of the IFAC Conference of Manufacturing, Modeling Management and Control, Athens, Greece, Oct, 2004; 21-22

12. Wilma P, Giovanni M. Manufacturing Signature for Tolerance Analysis. J Comput Inf Sci Eng. 2015; 15(2): 021005

13. Corrado A, Polini W, Moroni G. Manufacturing signature and operating conditions in a variational model for tolerance analysis of rigid assemblies. Res Eng Design. 2017: 1-16

14. Yan X, Ballu A. Generation of consistent skin model shape based on FEA method. Int $\mathrm{J}$ Adv Manuf Technol. 2017: 1-14

15. Cai N, Qiao L. Rigid-Compliant Hybrid Variation Modeling of Sheet Metal Assembly with 3D Generic Free Surface. J Manuf Syst. 2016; 41: 45-64

16. Cai N, Qiao L, Anwer N. Unified variation modeling of sheet metal assembly considering rigid and compliant variations. Proc Inst Mech Eng Part B-J Eng Manuf. 2014; 229(3): 495507

17. Bahubalendruni M V A R, Biswal B B. A novel concatenation method for generating optimal robotic assembly sequences. Proc Inst Mech Eng Part C-J Eng Mech Eng Sci. 2017; 231(10): 1966-1977

18. Bahubalendruni M V A R, Biswal B B. A review on assembly sequence generation and its automation. Proc Inst Mech Eng Part C-J Eng Mech Eng Sci. 2016; 230(5): 824-838

19. Ghandi S, Masehian E. Assembly sequence planning of rigid and flexible parts. J Manuf Syst. 2015, 36:128-146

20. Zhang X D, Zhang C, Wang B, et al. Unified functional tolerancing approach for precision cylindrical components. Int J Prod Res. 2005; 43: $25-47$

21. Ni WH, Yao ZQ. Cylindricity modeling and tolerance analysis for cylindrical components. Int J Adv Manuf Technol. 2013; 64: 867-874

22. Henke RP, Summerhays KD, Baldwin JM, et al. Methods for evaluation of systematic geometric deviations in machined parts and their relationships to process variables. Precis Eng. 1999; 23: 273-292

23. Madden M, Aketagawa M, Kumagai T et al. Concurrent measurement method of spindle radial, axial and angular motions using concentric circle grating and phase modulation interferometers. Meas Sci Technol. 2014; 25:
094005

24. Chien AY. Approximate harmonic models for roundness profiles with equivalent energy mean square values. Wear. 1982; 77: 247-252

25. Sawabe M, Fujimuma N, Sata T. Influence of radial motion on form error of workpiece in turning. Ann CIRP. 1978; 27: 505-509

26. Rahman M, Venkatesh VC. Effect of clamping conditions on chatter stability and machining accuracy. CIRP Ann-Manuf Technol. 1985; 34: 339-342

27. Rao BC, Shin YC. A comprehensive dynamic cutting force model for chatter prediction in turning. Int J Mach Tools Manuf. 1985; 39: 1631-1654

28. Sadílek M, Dubský J, Sadílková Z, et al. Cutting forces during turning with variable depth of cut. Perspect Sci. 2016; 7: 357-363

29. Stavropoulos P, Papacharalampopoulos A, Vasiliadis E et al. Tool wear predictability estimation in milling based on multi-sensorial data. Int J Adv Manuf Technol. 2016; 82: 509521

30. Schindler S, Zimmermann M, Aurich J C, et al. Thermo-elastic deformations of the workpiece when dry turning aluminum alloysA finite element model to predict thermal effects in the workpiece. CIRP J Manuf Sci Technol. 2014; 7: 233-245

31. Qiao L, Wu J, Zhu Z, et al. Approach to the Deviation Representation of Non-ideal Cylindrical Surfaces Based on the Curvilinear Coordinate System. Procedia CIRP. 2016; 43: 17-22

32. Vermeille H. Computing geodetic coordinates from geocentric coordinates. Journal of Geodesy. 2004; 78(1-2): 94-95 